\title{
LOS PREDICADOS ESTATIVOS Y LA EVIDENCIALIDAD: UN ANÁLISIS DESDE LA TEORÍA DE LOS BLOQUES SEMÁNTICOS
}

\section{STATIVE PREDICATES AND EVIDENTIALITY: AN ANALYSIS WITH THE SEMANTIC BLOCKS THEORY}

\section{SILVIA RAMÍREZ GELBES}

Universidad de San Andrés. Buenos Aires, Argentina.

Universidad de Buenos Aires. Buenos Aires, Argentina.

sgelbes@udesa.edu.ar

\section{RESUMEN}

En este trabajo se busca reconocer la existencia de dos clases de estativos -los estativos puros y los delimitados-, a partir de la relación diferenciada que cada grupo tiene con la evidencialidad, entendida ésta como el significado relativo a la fuente de información transmitido por el semantismo de ciertos verbos. Para hacerlo, se emplean las herramientas provistas por la Teoría de los Bloques Semánticos, específicamente en lo que se refiere a las argumentaciones externas. Según se observa, los predicados estativos delimitados siempre presentan una argumentación externa que involucra un predicado evidencial, en tanto los estativos puros no lo hacen.

Palabras clave: Semántica, estativos puros, estativos delimitados, argumento externo.

\section{ABSTRACT}

This paper seeks to recognize the existence of two kinds of stative verbs -pure statives and bounded ones- according to the differentiated relationship each group has with evidentiality, which is described as the source of information transmitted by a semantic content in certain verbs. To do this, the tools provided by the Semantic Blocks Theory are used, specifically in regard to the external argument. As shown, bounded stative predicates always have an external argument involving an evidential predicate, while pure statives do not.

Keywords: Semantics, pure statives, bounded statives, external argument.

Recibido: 06.09.2012. Aceptado: 05.01.2013. 


\section{INTRODUCCIÓN}

C n los últimos años, la aspectualidad ${ }^{1}$-entendida como el "modo de acción", el "carácter", la "naturaleza", la "índole" o la "cualidad de la acción o del proceso" propia de la situación denotada por el predicado- ha concitado el interés de filósofos y lingüistas como Vendler (1967), Comrie (1976), Mourelatos (1978), Dowty (1979), Bach (1986), Lyons (1997), Tenny (1989), Verkuyl (1993), Egg (1995), Morimoto (1998), Miguel Aparicio (1999), Marín (2000) y Filip (2005), entre muchos otros. Gracias a estos estudios, los predicados fueron tradicionalmente clasificados en estativos y dinámicos, pero sólo los dinámicos parecen haber merecido análisis más profundos y exhaustivos. En efecto, los estativos son aún considerados por muchos un gran grupo homogéneo de predicados, sin subdivisiones internas.

Por nuestra parte, hemos mostrado en otros trabajos (Ramírez Gelbes, 2008a, 2008b, 2008c, 2010, entre otros) que sí existen subdivisiones internas al grupo de los estativos. En este sentido, encontramos dos grupos de predicados estativos: los puros -del tipo de ser y de saber-y los delimitados -del tipo de estar y de conocer-. Y uno de los criterios que nos permiten reconocer la pertenencia de un estativo a uno u otro grupo es su comportamiento en relación con la evidencialidad. Como pretendemos probar aquí con la ayuda de la Teoría de los Bloques Semánticos (TBS), los estativos delimitados se relacionan discursivamente con los evidenciales, pero los estativos puros, no. Para decirlo de otro modo, tanto estar + adjetivo como conocer -y también estar en, resulta que y querer-contienen en su semantismo algún rasgo identificable con la noción de evidencialidad, dado que en una de sus argumentaciones externas estructurales contienen un elemento que sólo se verifica en términos de evidencia.

Para comprobarlo, en este trabajo, definiremos en primer lugar el concepto de evidencialidad; en segundo lugar estableceremos brevemente los lineamientos de la TBS; y en tercer y último lugar nos ocuparemos de relacionar las nociones de evidencialidad con los predicados estativos.

\section{LA EVIDENCIALIDAD}

Tema que ha suscitado, sobre todo en los últimos tiempos, un alto interés para los

\footnotetext{
${ }^{1}$ A los fines de la interpretación apropiada de nuestro planteo, debemos dejar en claro que la aspectualidad es un fenómeno que ha recibido distintos nombres en la literatura lingüística: Aktionsart (Comrie 1976, Hinrichs 1986; entre otros), aspecto léxico (Morimoto, 1998; Miguel Aparicio, 1999, entre otros) y también aspecto predicativo (González, 2003). En este trabajo seleccionamos el término aspectualidad (usado también por Verkuyl, 1993) para referirnos a la condición concebida como modo de acción o como tipo de situación a la que hace referencia el predicado.
} 
investigadores, la evidencialidad fue considerada al principio en relación con las lenguas amerindias, pero su estudio se ha extendido en la actualidad a las lenguas europeas (para un tratamiento general, ver Chafe y Nichols, 1986; Willet, 1988; Guentchéva, 1996; Aikhenvald, 2004, entre muchos otros; en español, ver Reyes, 1994; Bermúdez, 2005; López Ferrero, 2005, entre otros).

Para Reyes (1994), el evidencial es un tipo de significado transmitido por ciertas formas del verbo y por algunas construcciones adverbiales, que se producen cuando el hablante busca expresar que el conocimiento de lo que se dice no procede de la experiencia directa del hablante sino, antes bien, de una experiencia indirecta. López Ferrero (2005), por su parte, sostiene que la evidencialidad en sentido estricto consiste en la expresión de la forma como el conocimiento ha sido adquirido. Y en términos de Bermúdez (2005), el evidencial o marcador evidencial es la forma lingüística específica cuyo significado es una referencia a la fuente de la información.

Siguiendo entonces a Bermúdez, diremos que, dado que la evidencialidad es el dominio semántico relacionado con la fuente de la información expresada en un enunciado, las posibilidades son varias. De hecho, el hablante puede haber tenido contacto directo (visual o de otro tipo) con la situación descripta, haber tenido contacto no con la situación misma pero sí con indicios que apuntan hacia esa situación o haber recibido información de una tercera persona; y todo esto determina que la evidencia sea directa o indirecta (razonada o transmitida).

Pero también podemos verlo de la siguiente manera (Bermúdez, 2005: 6):

\begin{tabular}{|c|l|l|l|}
\hline \multirow{2}{*}{$\begin{array}{c}\text { TIPO DE } \\
\text { EVIDENCIA }\end{array}$} & directa & sensorial & $\begin{array}{l}\text { visual } \\
\text { auditiva } \\
\text { otros sentidos }\end{array}$ \\
\cline { 3 - 4 } & & endofórica & \\
\cline { 3 - 4 } & \multirow{2}{*}{ indirecta } & referida & $\begin{array}{l}\text { segunda mano } \\
\text { tercera mano } \\
\text { folklore }\end{array}$ \\
\cline { 3 - 4 } & & inferida & $\begin{array}{l}\text { inferencia } \\
\text { razonamiento }\end{array}$ \\
\hline
\end{tabular}

En relación con la evidencia directa y de acuerdo siempre con Bermúdez, hay dos tipos de evidencia: la sensorial y la endofórica. La sensorial se relaciona con la evidencia obtenida por medio de los sentidos y está representada en español por medio de algunos predicados de percepción, como ver el arco iris u oír el tema favorito. 
La evidencia directa endofórica, por su parte, se refiere a casos en los que el hablante describe entidades inaccesibles a los sentidos, como los deseos, las intenciones y los estados mentales en general. Por eso dice Bermúdez que se la encuadra en:

...aquellas situaciones en las que la evidencia sensorial es imposible, pero en las que el hablante aún aduce evidencia directa. Ejemplos de este tipo de evidencia serían casos como tengo sed, quiero irme, conozco la solución al problema, etc. (2005: 6).

La evidencia indirecta, a su vez, también la analiza el autor en dos subgrupos: la evidencia indirecta referida o transmitida, habitualmente llamada citativa, y la evidencia indirecta inferida.

La evidencia indirecta referida se relaciona con los casos en los que el hablante no tiene acceso ni a la situación ni a indicios de la situación, sino que la información le ha sido transmitida por otra persona, por ejemplo, el condicional de rumor, que se ve en (1), o el imperfecto citativo de (2), que Reyes (1994) interpreta como un discurso indirecto encubierto.

(1) 17 de marzo de 2009 13:00, por eLeVe. El Gobierno haría retoques a las retenciones (en línea). Disponible en http://www.ele-ve.com.ar/spip.php?article9502.

(2) - ¿Qué tal sigue Ana?

- Mejor, me parece. No la vi, porque cuando llegué, dormía. Pero había comido algo y tenía menos fiebre.

Por último, la evidencia indirecta inferida se refiere a casos en los que el hablante no tiene acceso directo a la situación descripta, pero sí posee contacto directo con huellas o rastros de esa situación que le permiten inferir lo que ocurre o ha ocurrido (inferencia) o conoce algo que le permite deducir que es probable que la situación se dé o se haya dado (razonamiento). Un ejemplo de huella es (3), mientras un ejemplo de razonamiento es (4).

(3) Llueve [porque vemos que Juan trae el paraguas mojado].

(4) Probablemente debamos llevar ropa abrigada a París Lporque es diciembre y allá es invierno].

Hasta aquí, entonces, hemos considerado sumariamente el fenómeno de la evidencialidad; necesitamos ahora una guía que nos permita relacionarlo con los predicados estativos. Es por ello que, en lo que sigue, nos ocuparemos de la Teoría de los Bloques Semánticos. 


\section{LA TEORÍA DE LOS BLOQUES SEMÁNTICOS}

La semántica argumentativa que se presenta en L'argumentation dans la langue (1983) de Jean-Claude Anscombre y Oswald Ducrot rompe con algunas expectativas propias de los estudios sobre argumentación, basados en la lógica o en la retórica. Su propuesta se centra en la semántica lingüística y afirma la existencia de una argumentatividad inherente a la lengua: la argumentación forma parte de los propios enunciados del discurso como integrante de su estructura lingüística y, por ello, esos enunciados están dirigidos hacia ciertos tipos de conclusión y no hacia otros. Dicho de otro modo, los enunciados no pueden ser empleados sin que el discurso se oriente hacia una determinada interpretación, por el simple hecho de que la lengua en sí misma posee una orientación argumentativa. Como los propios autores afirman:

Para nosotros, [la argumentación] es un rasgo constitutivo de numerosos enunciados el que no se los pueda emplear sin pretender orientar al interlocutor hacia un tipo de conclusión (por el hecho de que se excluye otro tipo de conclusión) (Anscombre y Ducrot, 1994: 48).

Según Anscombre y Ducrot (1994), hay cuatro etapas en el estudio de la argumentación: el descriptivismo radical, el descriptivismo presuposicional, la argumentación como un constituyente de la significación y la argumentatividad radical. Las dos primeras etapas, sostienen, son previas a su propuesta.

La primera etapa consiste en representar la lengua y la argumentación como si constituyeran instancias separadas, tal cual fue planteado por la tradición retórica. De acuerdo con esta postura, el contraste entre (5) y $(6)^{2}$ radica en que (6) designaría un lapso más breve que el primero, lo que aparecería como consistente con las nociones de "carácter permanente" y "carácter transitorio" de los predicados de ser y de estar, respectivamente, provistas por algunas descripciones al uso ${ }^{3}$.

(5) Ana es flaca.

(6) Ana está flaca.

En la segunda etapa, la filosofía analítica se ha ocupado de explorar ciertas propiedades semánticas de la lengua, especialmente la distinción entre contenidos afirmados y presupuestos. En alguna medida, este estadio (especie de reajuste del

${ }^{2}$ Ofrecemos aquí ejemplos con ser y estar-que son dos de los casos considerados en este trabajo-, pero advertimos que Anscombre y Ducrot no se ocuparon del contraste entre estos predicados.

${ }^{3}$ Aunque Anscombre y Ducrot no lo consideraron, en absoluto, desde la perspectiva de la argumentación, esta de permanente y transitorio es la explicación propuesta por autores como Bello (1945), Ragucci (1956) o Porroche Ballesteros (1988), entre otros. 
primero) estipula que el valor semántico de las frases se divide en valor afirmado y valor presupuesto. En tanto las informaciones provistas por los enunciados pueden estar afirmadas o presupuestas, los encadenamientos argumentativos conciernen exclusivamente a las informaciones afirmadas. Por esa razón, si se analizaran las diferencias entre (5) y (6) desde la perspectiva de este segundo estadio, podría decirse que, mientras en Ana es flaca se afirma la flacura y se presupone la existencia, en Ana está flaca se afirma la existencia y se presupone la flacura, lo que daría como resultado la conclusión de que en (5) la condición de ser flaca de Ana es aceptada como una cualidad mientras que en el segundo caso (6) es aceptada como un estadó.

El tercer estadio, el que corresponde propiamente a la versión inicial de la Teoría de la Argumentación en la Lengua o TAL (Anscombre y Ducrot, 1983), consigna la existencia de operadores argumentativos en la estructura misma de las frases. Eso significa que, junto a los valores descriptivos, la teoría reconoce la presencia de ciertos valores argumentativos independientes que se evidencian en determinadas expresiones. Es a partir de este estadio cuando se empieza a reconocer que las propias expresiones exigen que sus enunciados sean usados argumentativamente y que lo sean con una determinada dirección. En consecuencia, es en este estadio cuando puede considerarse que la diferencia entre (5) y (6) consiste en que ser y estar funcionan como operadores aspectuales que determinan perspectivas diferentes para la aceptación de uno u otro enunciado: el primero, Ana es flaca, ofrece una visión que podría llamarse "de norma general", mientras que el segundo, Ana está flaca, provee una visión que podría considerarse "de norma individual"s.

El cuarto estadio corresponde a la posición denominada argumentatividad radical, versión estándar de la TAL, y se pone de manifiesto en la utilización sistemática de la noción de topos. De acuerdo con esta posición, los "hechos" descriptos por las expresiones ya no son otra cosa que la cristalización de movimientos argumentativos. En efecto, hasta el tercer estadio de los estudios sobre la argumentación, se conserva la idea de que, más allá de la existencia de ciertos componentes inherentemente argumentativos -los operadores argumentativos-, existen en la lengua expresiones puramente informativas, es decir, expresiones que remiten al mundo extralingüístico. Por el contrario, en el cuarto estadio se abandona esta idea y se propone la existencia de una argumentatividad inherente a la lengua en su totalidad.

El encadenamiento discursivo, para este último estadio, va de un enunciado

\footnotetext{
${ }^{4}$ Buscando una analogía entre los ejemplos de (5) y (6) y esta propuesta, creemos que, aunque de ningún modo lo plantearon desde la argumentación, esta explicación de cualidad frente a estado aparece representada en Alonso y Henríquez Ureña (1946).

${ }^{5}$ Esta concepción es la propia de Falk (1979), quien no vio el fenómeno desde el punto de vista de la argumentación sino desde un punto de vista exclusivamente pragmático.
} 
argumento a un enunciado conclusión por medio de la aplicación de principios generales llamados topoi. Así, si de un enunciado $A$ se concluye un enunciado $B$, es porque $A$ presenta al hecho $H$ (configurado por $A$ ) de modo que vuelve legítima la aplicación de un topos (o de una cadena de topoi) que conduce a $B$. Para decirlo de una manera menos formalizada, se afirma que la significación de una expresión es el conjunto de topoi cuya aplicación autoriza la propia expresión al ser enunciada. Así, en una situación concreta, la elección de una expresión en lugar de otra significa seleccionar la explotación de unos topoi en lugar de otros. Esto es, el valor semántico de las expresiones reside en autorizar y más aún en promover ciertos puntos de vista argumentativos.

Desde esta perspectiva, los predicados de la lengua son descriptos como si fueran haces de topoi. Eso significa que cada expresión no orienta hacia una única conclusión de manera privativa sino que, antes bien, despliega una serie de posibilidades argumentativas que, eso sí, no son infinitas. Por ello, comprender una expresión de la lengua significa ser capaz de reconocer la relación de esa expresión no con una única orientación sino con una serie de orientaciones posibles, lo que transforma el campo léxico en una especie de campo tópico (Bruxelles, Ducrot y Raccah, 1993).

Esta idea debe verse dentro de una óptica claramente estructuralista, lo que determina que cada una de estas orientaciones tópicas tenga valor exclusivamente en términos de sus relaciones con todas las demás (cf. Saussure, 1955). De todos modos, debe quedar claro que no se espera ni que todos los individuos de la comunidad compartan exactamente los mismos campos tópicos ni que un sujeto particular use siempre el mismo campo tópico en contextos similares.

Sea como fuere, este estadio declara no sólo que no hay frases informativas en la lengua sino que ni siquiera existe un componente informativo en la significación de las frases. A lo sumo, los usos (pseudo)informativos son derivados de un componente que es más profundo en la lengua y que es el componente argumentativo. En esta perspectiva, entonces, la diferencia entre Ana es flaca y Ana está flaca podría ser la consideración de que el primer enunciado reclama un topos del tipo "si se es ADJETIVO (aquí, flaca), cuanto menos ligado a las coordenadas tempoespaciales, más se es ADJETIVO (aquí, flaca)" mientras que el segundo enunciado reclama uno como "si se es ADJETIVO (aquí, flaca), cuanto más ligado a las coordenadas tempoespaciales, más se está ADJETIVO (aquí, flaca)" ${ }^{\text {. }}$

A lo afirmado por Anscombre y Ducrot (1983), agregaremos un último estadio en los estudios sobre la argumentación. Este estadio se corresponde con el desarrollo actual de la TAL, avance que ha dado en llamarse Teoría de los Bloques Semánticos (TBS). En esta etapa, el avance consiste en abandonar toda noción de elemento extralingüístico -como pueden ser los topoi-para centrarse en una

${ }^{6}$ Cf. Ramírez Gelbes (2004). 
propuesta que sólo recurre a nociones intralingüísticas. En efecto, el concepto de topos aparece reemplazado por el de argumentaciones, que son definitivamente lingüísticas y que se estipulan no ya fuera de la situación concreta de discurso sino en la situación de discurso particular. En efecto, no se habla más de un pasaje que va de antecedente a conclusión provisto por un topos externo sino, antes bien, de una interdependencia semántica entre antecedente y consecuente.

En efecto, para la TBS, creación de Marion Carel en su tesis doctoral de 1992, la argumentación inherente a la lengua se ve manifestada por medio de encadenamientos argumentativos. Así, si tenemos la frase está flaca, los encadenamientos que componen su sentido pueden tener que ver con enunciados del tipo de (7) o de (8), en los que la noción de flacura se relaciona con la idea de algo deseable, algo positivo y valorado. Pero también pueden tener que ver con enunciados del tipo de (9) o de (10), en los que la idea de flacura se conecta con un valor negativo, indeseable.

\section{(7) Está haciendo dieta, por lo tanto está flaca.}

(8) Está flaca, sin embargo no la contrataron para el desfile.

(9) La bebé come bien, sin embargo está flaca.

(10) La caballada está flaca, así que la vamos a vender muy mal.

Para decirlo de otro modo, el está flaca en (7) y (8) se relaciona con un estado que se busca y que permite acceder a una situación deseada. Por el contrario, el está flaca en (9) y (10) se refiere a un estado contrario a lo deseado y puede provocar un resultado negativo.

Esto viene a decir que un enunciado del tipo A por lo tanto $\mathrm{B}$ es argumentativo porque convoca principios o estereotipos que no son asociaciones de conceptos independientes ni están en la relación que liga argumento y conclusión. En definitiva, la argumentación no es un proceso de justificación por el cual el argumento deba fundamentar la conclusión y tampoco es un proceso que aparezca validado por un tercer elemento -como pasaba con el topos-: el argumento y la conclusión son representaciones unitarias que, al unirse, constituyen el sentido propio del enunciado realizado por los encadenamientos argumentativos.

Por ello, un enunciado del tipo (7) a (10) provee sentido sólo en la medida en que ambos segmentos unidos por el conector están juntos. Vistas así, las argumentaciones no tienen un "apriorismo argumentativo" que las sustente, dependen absolutamente del bloque semántico que queda definido por la presencia conjunta del argumento y la conclusión. La verdad del discurso no tiene que ver con la realidad extralingüística sino con el discurso mismo, con la verdad que se construye por medio del lenguaje. Argumentar consiste en reunir bloques léxicos y pretender ser coherente con ellos.

En otras palabras, el sentido no se encuentra en la inferencia argumentativa 
dada por el pasaje del argumento a la conclusión, sino que se construye estrictamente en la relación de dependencia semántica de ambos segmentos reunidos para conformar un todo.

Generalizando, entonces, las expresiones no tienen un sentido fijo, independiente del discurso en el que ocurren: su sentido viene determinado por el tipo de discursos que se le asocian, discursos evidenciados en los encadenamientos ejemplificados por los enunciados de (7) a $(10)^{7}$. O, en pocas palabras, el sentido de una expresión está dado por los encadenamientos discursivos, de orden estrictamente lingüístico, que esa expresión autoriza. Esos encadenamientos discursivos están representados de la siguiente manera:

\section{$A$ CONECTOR $B$}

Esto significa que el encadenamiento discursivo está constituido por dos segmentos, $A$ y $B$ respectivamente, que se relacionan por medio del conector. Por esa razón, si tomamos los siguientes enunciados:

Estar flaca

Ser contratada para el desfile

hay distintas relaciones argumentativas que se pueden dar entre ellos, como (11) o (12), a saber:

(11) Ana, que es modelo, está flaca, por lo tanto será contratada para el desfile.

(12) Ana, que es modelo, no está flaca, por lo tanto no será contratada para el desfile.

Tanto en (11) como en (12), desfile evoca el desfile de modas al uso a comienzos del siglo XXI, en el que las modelos de pasarela deben ser muy espigadas. Pero también pueden darse (13) y (14), y en ese caso desfile evoca un tipo de desfile distinto, un desfile en el que la flacura no es aceptable.

\section{(13) Ana, que es modelo, está flaca, por lo tanto no será contratada para el desfile.}

\footnotetext{
${ }^{7}$ Debe observarse que el sentido de las expresiones, para la TBS, no se reduce a los discursos en los que esas expresiones se insertan y que la teoría denomina argumentaciones externas (AE). Existe un segundo tipo de discursos que contribuyen al sentido de la expresión: las llamadas argumentaciones internas (AI). Si no nos ocupamos de las $\mathrm{AI}$ en este trabajo es simplemente porque aquí relacionamos el contenido evidencial con las $\mathrm{AE}$, pero propondremos una brevísima descripción de este tipo de argumentaciones. Las AI son encadenamientos que parafrasean la expresión sin contenerla y actúan como definiciones (al modo de las definiciones de diccionario) de la expresión dentro del marco de esta teoría. Así, la AI de flaca podría pensarse como volumen esperable SE magro, desde el momento en que este encadenamiento puede funcionar como una definición (o expresión parafrástica) del término.
} 
(14) Ana, que es modelo, no está flaca, por lo tanto será contratada para el desfile.

Estas posibilidades muestran que la propia relación argumentativa da sentido a las expresiones determinando una interdependencia semántica: la misma expresión, desfile, refiere en un caso a un tipo de desfile en el que se buscan modelos flacas y, en el otro, a un desfile en el que las modelos flacas son rechazadas ${ }^{8}$.

Ahora bien, la relación argumentativa de dos elementos (como está flaca y desfile en los ejemplos anteriores) puede hacerse efectiva por medio de dos conectores: POR LO TANTO (PLT) y SIN EMBARGO (SE). Debe entenderse, eso sí, que ambos conectores son conceptuales o abstractos y se realizan por medio de distintos marcadores o estructuras (como se ve en Está flaca: la contratarán para el desfile o No la contratarán para el desfile porque está flaca, por ejemplo). Cuando se trata de una relación con el primer conector -i. e. POR LO TANTO-, hablamos de un encadenamiento normativo; cuando se trata de una relación con el segundo conector -i. e. SIN EMBARGO-, hablamos de un encadenamiento transgresivo.

En consecuencia, dados todos los encadenamientos posibles, parece claro que algunos se encuentran emparentados, como es el caso de a), b), c) y d) ${ }^{9}$ :
a) $A$ PLT B
b) $N e g-A S E B$
c) $A S E N e g-B$
d) Neg-A PLT Neg-B

En nuestro ejemplo, dados "estar flaca" (A) y "ser contratada para el desfile" (B), podemos derivar los siguientes ejemplos a), b), c) y d):

a) Ana está flaca, por lo tanto la contratarán para el desfile.

b) Ana no está flaca, sin embargo la contratarán para el desfile.

c) Ana está flaca, sin embargo no la contratarán para el desfile.

d) Ana no está flaca, por lo tanto no la contratarán para el desfile.

En todos estos casos, como en (11) y en (12), el sentido de está flaca es el de una condición positiva, deseable, en tanto el sentido de desfile es el de desfile en el cual las modelos son flacas. Y puesto que la interdependencia semántica es la misma, se puede decir que las cuatro expresiones forman parte del mismo bloque.

\footnotetext{
${ }^{8}$ Este fenómeno pone en evidencia que el tipo de relación que se establece, una relación de tipo discursivo, no remite a los razonamientos propios de la lógica clásica porque, si lo hicieran, estaríamos hablando de contradicciones. Como se recordará, en la lógica clásica, el valor de los segmentos es independiente de las relaciones que se establecen entre ellos. En suma, la TBS sostiene que la lógica discursiva tiene condiciones diferentes de las propias de la lógica filosófica.

${ }^{9}$ Cada una de estas posibilidades es llamada aspecto.
} 
Pero sabemos, además, que otros cuatro encadenamientos son posibles:
a) $A S E B$
b) Neg-A PLT B
c) $A$ PLT Neg-B
d) $N e g-A S E N e g-B$

encadenamientos que, para los enunciados correspondientes, aparecen representados en a'), b'), c') y d').

a) Ana está flaca, sin embargo la contratarán para el desfile.

b') Ana no está flaca, por lo tanto la contratarán para el desfile.

c) Ana está flaca, por lo tanto no la contratarán para el desfile.

d) Ana no está flaca, sin embargo no la contratarán para el desfile.

Y se ve que, en estas cuatro últimas realizaciones, al igual que en (13) y en (14), está flaca tiene el sentido de algo negativo, no deseable, en tanto que desfile tiene el sentido de un desfile en el que las modelos no son flacas. En consecuencia, las cuatro expresiones presentadas en a') a d') constituyen un bloque y ese bloque, a su vez, se distingue notoriamente del anterior.

Es claro, entonces, que la interdependencia semántica se repite en los cuatro aspectos del mismo bloque. $\mathrm{O}$, formulado a la inversa, el sentido idéntico de $A$ y $B$ en diversos encadenamientos (condición deseable de A para a), b), c) y d); condición indeseable de A para a'), b'), c') y d')) es evidencia de que esos encadenamientos pertenecen al mismo bloque.

Dado el ejemplo de a), entonces,

a) Ana está flaca por lo tanto la contratarán para el desfile.

se puede construir el siguiente bloque:

Neg-estar flaca SE ser contratada

estar flaca SE Neg-ser contratada

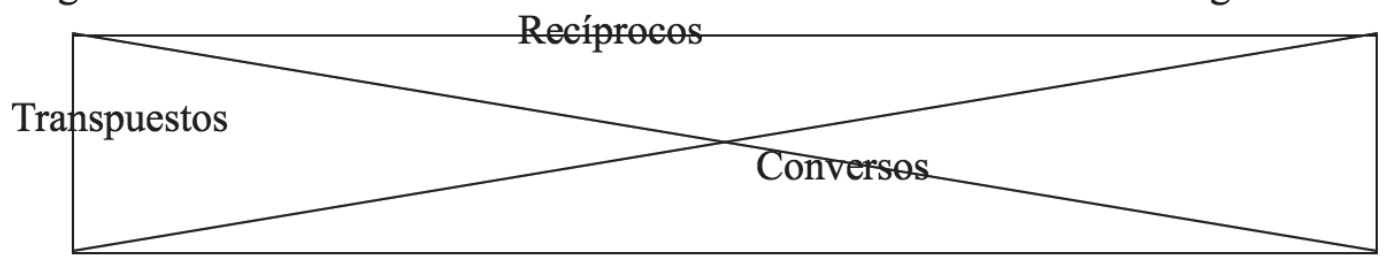

estar flaca PLT ser contratada

Neg-estar flaca PLT Neg-ser contratada 
A: estar flaca para ser contratada

$B$ : ser contratada

$B S_{I}$ : el desfile requiere modelos flacas

Pero hemos visto que también existe c') y, por consiguiente, otro bloque:

c) Ana está flaca por lo tanto no será contratada

Neg-estar flaca SE Neg-ser contratada estar flaca SE ser contratada

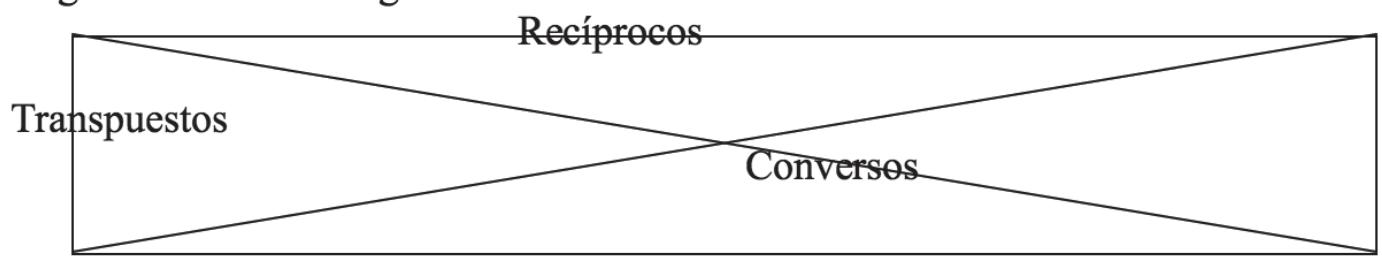

estar flaca PLT Neg-ser contratada

Neg-estar flaca PLT ser contratada

$A$ : estar flaca para no ser contratada

$B$ : ser contratada

$B S_{2}$ : el desfile requiere modelos no flacas

Ahora bien, la TBS afirma que el sentido de una expresión está dado por los discursos, de los cuales esa expresión forma parte y, al mismo tiempo, por los discursos de los que, sin formar parte, la parafrasean. Los discursos del primer tipo son llamados argumentaciones externas; los del segundo tipo, argumentaciones internas (ver nota 7).

Las argumentaciones externas (AE) de una unidad lingüística, como venimos diciendo, son los encadenamientos en los que esa expresión es o bien el origen o bien el término, lo que significa que la propia expresión siempre forma parte de sus argumentaciones externas. Al mismo tiempo, cada $\mathrm{AE}$ de una expresión está asociada a su converso, lo que significa que un aspecto de la $\mathrm{AE}$ de una expresión con uno de los conectores (por ejemplo, para el caso de (15)) siempre está asociado a un aspecto con el conector opuesto $+\mathrm{Neg}$ (siguiendo con el ejemplo, (16)); debe observarse, eso sí, que una $\mathrm{AE}$ nunca puede tener dos aspectos recíprocos (para (15), el caso de (17)), porque no puedo decir a un mismo tiempo A y Neg-A, o, lo que es igual, si estamos considerando el sentido de es linda sólo podemos tomar los discursos que contengan esa expresión y no los que la transformen en no es linda.

(15) Ana es linda por lo tanto está linda.

(16) Ana es linda sin embargo no está linda.

(17) *Ana no es linda por lo tanto no está linda. 
Finalmente, las AE se clasifican en estructurales y contextuales.

Las AE son estructurales cuando forman parte del sentido lingüístico de la entidad, o sea, están previstas por la lengua. Decimos que (15) es una AE estructural porque no admite la existencia de un segundo bloque.

(15) Ana es linda por lo tanto está linda.

(18) *Ana es linda por lo tanto no está linda.

De hecho, (16) puede verse representado en el segmento (19).

(19) Volvió a pensar en las cartas. Sonrió al ver la segunda, pues era más agradable releerla. Carmen te contaba que la profesora habia conversado con ella y "hasta me ha preguntado de ti. Me ha dicho: ¿Qué es de tu hermano Marcos? ¿Vuelve pronto? Ella es linda, hermanito, aunque seguro que tiene algún sufrimiento oculto; pero ahora desde que enterraron a la señorita Gertrudis (esa viejita medio loca y buena como una santa, ¿te recuerdas?) y celebraron su misa de nueve días, está más tranquila y más linda. Algunos dicen que ha viajado dos veces a la ciudad para pedir su traslado, pero son habladurias" (Prada Oropeza, Renato. 1979. Larga hora: la vigilia. México: Premia).

donde se verifica la fórmula:

ella es linda SE no estaba (tan) linda (mientras Gertrudis estaba viva) (es X SE Neg-está X)

Una lectura rápida del fragmento, sobre todo del encadenamiento marcado en negrita, podría conducir a una interpretación inaceptable: *es linda SE está linda. Pero no es eso lo que dice el fragmento: lo que hace este fragmento es manifestar el BS que proponemos en la fórmula es X SE Neg-está X.

En efecto, aquí se afirma "Ella es linda (esencialmente) pero (antes, cuando vivía Gertrudis) no estaba linda" -o es X SE Neg-está X-; y luego se agrega: "Ella no estaba linda antes pero ahora sí que está linda" -o Neg-está linda en T-1 SE está linda en $\mathrm{T} 0$-. Por ello, el primer segmento puede ser leído como "aunque es linda, concedo que mientras Gertrudis vivía no estaba demasiado linda” y el segundo como "antes no estaba linda pero ahora está linda". El primer segmento, entonces, patentiza la existencia de ese encadenamiento transgresivo -representante del BS del que hablamos- es linda SE Neg-está linda, bloque que es estructural, porque representa un BS único al rechazar la existencia de un $\mathrm{BS}_{2}{ }^{*}$ es linda PLT Neg-está linda.

Por el contrario, la $\mathrm{AE}$ es contextual cuando es la situación de discurso la que la vincula con la entidad; es decir que para aceptar la conversa de la contextual, 
debo cambiar la situación. Tal el caso de (20) y (21). En (20), se necesita una empleada agraciada, por ejemplo, para cumplir la tarea de recepcionista, la persona que yo puedo tomar como primera imagen que se tiene de la empresa. En (21), en cambio, se trata de un trabajo en el que necesito una mujer que no sea linda, por ejemplo, una actriz que debe hacer el papel de un personaje no lindo.

(20) Ana es linda por lo tanto le daré el trabajo.

(21) Ana es linda por lo tanto no le daré el trabajo.

Así, (20) aparece representado en (22) donde la AE desplegada se puede corresponder con Ana no es bonita SE le daré el trabajo. (De hecho, la aclaración entre paréntesis de que es poco probable que una secretaria tenga mala presencia y mala letra hace referencia al estereotipo de las secretarias atractivas y prolijas).

(22) Otro ejemplo lo vemos en el uso del teléfono. Cuando usted habla por teléfono solo dispone de la voz; faltan los gestos. Por ejemplo, aunque una secretaria sea muy bonita $y$ despliegue gracia al andar, escriba con buena letra y sea muy servicial, si su voz suena desagradable cuando usa el teléfono, puede parecer una mujer de aspecto horrible. $O$ al revés, transmitir la imagen de una mujer muy bonita gracias a una voz maravillosa, aunque tenga mala presencia y mala letra (esto último es poco probable) (Ruiz Orbegoso, M. 2003. Sugerencias para aprender a exponer en público. (en línea). Disponible en: http:// www.librosenred.com/libros/sugerenciasparaaprenderaexponerenpublico. html. Consulta: 26.03.2013).

Lo que plantea el locutor es: "Aunque sea bonita, si su voz suena desagradable, parece una mujer de aspecto horrible, en consecuencia, no le daré el trabajo", discurso en el que aparecen representados, a la vez, dos aspectos:

a. "Aunque sea bonita no le daré el trabajo": es linda SE Neg-le daré el trabajo

b. "Parece una mujer de aspecto horrible por lo tanto no le daré el trabajo": Neges linda PLT Neg-le daré el trabajo

Pero a continuación sostiene: "Si tiene una voz maravillosa, es linda (para quien la escucha del otro lado del teléfono) aunque tenga mala presencia y mala letra”, discurso en el que se representan los dos aspectos restantes del mismo BS:

c. "Aunque tenga mala presencia le daré el trabajo": Neg-es linda SE le daré el trabajo

d. "Si da la imagen de una mujer muy bonita, le daré el trabajo": es linda PLT le daré el trabajo 
De este modo, la coherencia del razonamiento que percibe el destinatario está dada por la presentación de los argumentos como pertenecientes al mismo bloque semántico, es decir, a la misma argumentación.

(21), por su parte, aparece bien representado en (23), donde no se busca ya representar la imagen de la empresa (como podía pasar en (22)) sino evitar la mezcla del trabajo con "las tentaciones" o "la diversión".

(23) "Me gusta Mary porque es una profesional, una secretaria ejecutiva de verdad, en la que puedo confiar. Hay cuestiones que le abandono ciegamente. Y además - habia sonreido- me gusta porque es vieja, flaca y sin gracia. No quiero tentaciones cerca". Y habia vuelto a sonreir más ampliamente y con picardia. "No soy partidario de mezclar los negocios con los 'asuntos" e hizo un guiño de inteligencia a Adrián, que si hubiera sabido idiomas habría entendido el juego de palabras. "No hay nada más importante que una secretaria eficaz, es más importante que el mejor de los jefes. Guárdeme el secreto, pero para mi es más valiosa Mary que Morales. No le diga nada porque es muy celoso y además tiene tendencia a las depresiones", y habia lanzado la carcajada. "Hágame caso, Larralde, no elija nunca una secretaria linda") (Andrade, J. 1993. Un solo dios verdadero. Madrid: Anaya).

En efecto, en (23) se presenta un bloque semántico distinto del anterior, porque el locutor afirma:

a. "Si la secretaria es linda, no le dé el trabajo": es linda PLT Neg-le daré el trabajo

b. "Mary no es linda por lo tanto le di el trabajo": Neg-es linda PLT le daré el trabajo

Así, el bloque que despliega (23) puede resumirse como Neg-es linda PLT le daré el trabajo mientras que (22), ya lo hemos visto, despliega Neg-es linda PLT Neg-le daré el trabajo. Y son estas dos argumentaciones diferentes -que, obviamente, aparecen evidenciadas en discursos distintos y que representan un $\mathrm{BS}_{1}$ y un $\mathrm{BS}_{2}$ - las que determinan que hablemos aquí de $\mathrm{AE}$ contextuales.

En definitiva, la AE es estructural cuando los dos miembros del encadenamiento sólo pueden componer un bloque (el segundo bloque no resulta razonable), en tanto que es contextual cuando esos miembros pueden componer, aceptablemente, dos bloques.

\subsection{La AE de estar y la evidencia}

Hemos afirmado en otro lugar (Ramírez Gelbes, 2010) que una AE estructural a la 
izquierda de estar (es decir, una en la que estar + adjetivo es el término) es:

AE de estar: se ve X PLT está X

Neg- se ve X SE está X

como muestra la implausibilidad de:

AE de estar: ${ }^{*}$ Neg-se ve X PLT está X

* se ve X SE está X

Este análisis nos permite releer a Bello (1945: 335), quien señala que la oposición entre las construcciones con estar y las construcciones con ser en los mismos contextos se relaciona con el hecho de que, con ser, el sintagma denota "la impresión real o figurada que el agente hace en el objeto", como se ve en (24), donde no resulta necesaria la intervención de la experiencia directa.

(24) La nieve es fría.

Ese mismo sintagma en un predicado de estar denota el estado que es la consecuencia de la impresión real o figurada que el agente hace en el objeto, como se constata en (25).

\section{(25) El agua está fría.}

Dicho de otra manera, el sintagma adjetivo ocurre con estar cuando el hablante presenta su discurso como dependiente de la experiencia concreta. Y es lo que también afirma Gili y Gaya, que define el uso de estar como que el hablante plantea un juicio que depende de su propia experiencia previa: "Empleamos estar en los juicios que dependen inmediatamente de nuestra experiencia (...); para decir que Aquella nieve está fría necesito tocarla ahora" (Gili y Gaya, 1948: 57 y 58).

Desde nuestro punto de vista y dentro de la perspectiva de la TBS, el presupuesto (o segmento a la izquierda) de una de las AE estructurales de estar + adjeti$v o$, en efecto, evidencia lo que Bello y Gili y Gaya expresan a su modo:

AE de estar: se ve (se siente) PLT está

*Neg-se ve (se siente) PLT está

Como se observa en esta $\mathrm{AE}$, es el propio discurso el que determina que la presuposición consiste en haber tenido contacto directo con la situación. Por eso, sostenemos, estar + adjetivo expresa una relación con una evidencia directa, que es lo que podemos ver, o lo que podemos sentir, tal como queda claro en (26), donde 
el estar espesa de la preparación surge de la experiencia de tener más dificultad al removerla, de la textura y del color, es decir, de la evidencia sensorial directa. Y es ese el sentido propio del estar que hemos descripto como evidencial ${ }^{10}$. O en (27), donde queda claro que el locutor se expresa después de ver el departamento, hecho resaltado por la sorpresa de lo que ve y que se verbaliza por medio de caray. Esa visión, patentizada aquí por la interjección, le permite expresar está lindo.

(26) En un mortero se hace un picado de ajos, con perejil y sal, vertiéndolo en el recipiente. Al estar todo bien cocido se saca caldo, se cuela, y con él se hacen los formigons. Para ello se deja enfriar el caldo y entonces se va echando harina y removiendo. Al estar totalmente disuelta se pone al fuego y se deja cocer. Cuando está espesa se retira (Vergara, A. 1981. Comer en el País Valencià. Madrid: Penthalon).

(27) Bajaron, entraron al edificio, subieron por el ascensor y pasaron al departamento de Patty.

- Caray, está lindo — dijo Diego.

Era un departamento grande, alfombrado, con vista al mar (Bayly, J. 1996. Los últimos días de La Prensa. Barcelona: Seix Barral).

Más aún, la argumentación de que los predicados de estar + adjetivo presentan el discurso como proveniente de una experiencia directa podría fundamentar la distinción del sentido de bueno en es bueno y está bueno en (28) y en (29). Así, en (28), es bueno remite a una condición homogénea que refiere a un carácter moral o funcional, una condición que se presenta como fuera de límites tempoespaciales (y es por eso que, en (28), el locutor afirma que "él está perdido eventualmente" a causa del vino, al modo de es bueno SE Neg-está bueno en este momento a causa del vino). Por el contrario, en (29), está bueno alude a la idea de ver, de constatar, de tener una prueba y, por eso, el locutor sólo puede afirmarlo después de que el sujeto en cuestión se ha dado vuelta, es decir, después de que lo ha visto.

(28) JOSEFA. - -Ay Padre! (Suspirando compasivamente.). Si él es bueno, bruto pero bueno. El vino, el maldito vino es el que lo lleva a la perdición (Gallego, C. 1990. Adelaida. Madrid: Marsó-Velasco).

(29) ...entonces nos asomamos en realidad a la puerta a ver, como a las siete de la mañana, porque habian llegado como a las cuatro de la mañana, y estaba él, como decir, allí en donde está la floristería, estaba él parado así con las manos en

${ }^{10}$ Desde luego, las mismas observaciones podrían hacerse en relación con estar cocido y estar disuelta que aparecen en el mismo ejemplo (26). 
la espalda, yo me acuerdo clarito como si lo estuviera viendo. Entonces, le digo: chica, pero él no voltea. Para que voltee para yo ver si es buen mozo, si me interesa $o$ no. En realidad no era feo, era un hombre muy buen mozo, muy igualito al hijo era. Entonces le digo yo: bueno, sí, chica, está bueno, pero ahi te lo dejo a ti (ORAL. CSHC-87. Entrevista 155. Venezuela).

Desde luego, esa constatación que pide estar puede también surgir de alguna forma de referencia citativa o de razonamiento, como es el caso de (30), en el que el locutor combina sus percepciones ("Asunción se ha modernizado, se han jerarquizado las plazas, se ha mejorado el tránsito, se han agilizado los trámites") con una especie de razonamiento que surge de la comparación ("yo conozco otros países"), y eso determina, entonces, una forma de evidencia un poco más indirecta, pero evidencia aun así.

(30) ...todos los barrios de Asunción se han modernizado, se ha jerarquizado las plazas, se ha mejorado el tránsito, y en cuanto a los pagos de servicio, también, uno viene por anticipado, pide, incluso ya en la mesa, la boleta y de abi ya tiene plazo de pagar un mes, si pasa, al otro mes tiene un pequeño recargo, pero es muy límite, o sea, que es muy variado. Sí, sí, es que lo calló bien, para mi que lo que faltó fue un poquito de concienciación a toda la ciudadanía. Es cosa que es muy moderno, el costo tiene su limite, y para mi que es una modernización en cuanto a parquimetro. La gente no quiere pagar impuesto, hay que ser claro, el Paraguayo no quiere pagar impuesto, se enoja cuando va al aeropuerto, paga una tasa, se va a la terminal, paga otra tasa. Para mi que está lindo, yo particularmente quiero, porque conozco otros paises y en otros paises no te perdonan nada, tanto Río para cualquier movimiento se paga por fichas, el sistema de fichas, incluso para llamar por teléfonos públicos de Río. Yo llamé a Asunción con una fichita que me costó una minima suma, yo hablé a Asunción. También acá en Paraguay se quiere estilar al próximo año, y ya desde un teléfono público hacer llamada para otro país (ORAL. Encuesta 27, Octavio Gómez Ocampos. Paraguay).

Es más, la propia condición de evidencial del predicado de estar se manifiesta también en las ocurrencias de $X$ estar en aunque, en este caso, como una AE estructural a la derecha, según muestra (31) por medio de un discurso que podríamos formalizar como estaba ahí SE no lo habíamos visto (está en X SE Neg-se ve).

(31) Hasta que al fin las cosas se cansaron y decidieron imponernos su voluntad de vivir. Recuerdo muy bien esa vez en que de pronto, a la mitad de una conversación de sobremesa, nos callamos al mismo tiempo como si hubiera pasado un ángel y 
descubrimos en medio de la mesa un vaso que jamás habiamos visto. Con esto no quiero decirte que apareció de milagro, no: estaba alli desde la mañana, y antes de eso estaba en la alacena y mucho antes en la tienda donde lo compramos. Pero nunca lo habíamos visto. "¿Te fijaste? -me preguntó Estefanía-. Parece un vaso". “De dónde habrá venido?”, le pregunté. "¿Qué querrá de nosotros?”, dijo ella (Del Paso, F. 1982. Palinuro de México. Madrid: Alfaguara).

En definitiva y dentro de la óptica de la TBS, los discursos que le están asociados a estar en por lo menos alguna de sus AE estructurales muestran que estar se vincula con la evidencialidad.

\subsection{La AE de conocer y la evidencia}

Para Rémi-Giraud y Le Guenn (1986: 250), el verbo connaître representa una especie de saber que "toca las cosas", a la manera de un saber de experiencia. Matte Bon (1995: 233 y ss.), por su parte, explica que "conocer algo es haber estado en contacto o haber tenido experiencia de ese algo sin que se haya convertido en una propiedad de quien lo conoce". Para nosotros, estas definiciones se ponen de manifiesto en una AE estructural de conocer:

AE de conocer: haber visto PLT conocer

${ }^{*}$ Neg-haber visto PLT conocer

De hecho, si afirmamos (32), estamos haciendo referencia a los datos efectivos que Juan presenta como muestra de su conocimiento, el conocimiento que tiene gracias a que, de un modo u otro, se ha enfrentado con la fuente de ese conocimiento, tiene una evidencia directa y la expresa concretamente. $Y$ así se ve en (33), donde se explicita esa fuente.

(32) Juan conoce la verdad.

(33) El fiscal le preguntó si sabía que en enero de 1992 Portland Iberia, del grupo Banesto, habia comprado un 101,7e de Isolux.

- Lo conozco porque me lo comenta el señor Romani — se escudó (Ernesto Ekaizer. 1996. Vendetta. Barcelona: Plaza y Janés).

En (33), el contraste entre el uso de saber por parte del fiscal y el uso de conocer por parte del interrogado pone en claro que este último quiere marcar que él tiene una evidencia - indirecta, citativa o referida, lo que "le comenta el señor 
Romani"- y que esa es la fuente de su conocimiento, no otra. Dicho en otras palabras y para comprender el comentario (se escudó), sostenemos que el locutor muestra que la fuente de su conocimiento son las palabras de otro, no el hecho de haber estado involucrado en el hecho o de haber participado del hecho: se trata, en efecto, de una evidencia indirecta citativa. Puede verse asimismo (34), donde la evidencia indirecta citativa se hace patente por la presencia del discurso referido representado por el condicional simple de apelarian.

(34) También se conocía que los chiricanos apelarían hasta las últimas consecuencias legales, una suspensión de 3 años. (El Siglo, 09/05/1997: "La selección juvenil de baloncesto parte hoy a las 5 de la tarde con destino...”).

Es más, en (35), el locutor plantea que la actitud del mensajero le brinda indicios -o le muestra una huella, en términos de Bermúdez (2005)- de lo que ocurre, es decir la fuente de su conocimiento es esa actitud de silencio. Por lo que, nuevamente, nos encontramos con una evidencia indirecta inferida como fuente de conocer.

(35) - Tendrás diez provincias si me dices que Antonio viene pisándote los talones. $O$ si me indicas que acuda corriendo a mis estancias, porque fue directamente a abrazar a sus hijos, tanto ansiaba conocerlos. Pero callas. Por tu silencio conozco que no llega Antonio. Entonces ¿qué mensaje traes? ¿Dice Antonio que aún ama a su reina? ¿O sólo quiere saber de sus dos príncipes? (Moix, T. 1993. No digas que fue un sueño. Barcelona: Planeta).

Pero no es necesario que conocer trate siempre de evidencias indirectas. En efecto, también encontramos casos que nos permiten hablar de una evidencia directa, como en (36), donde el locutor deja en claro que la fuente del conocimiento es definitivamente sensorial -los olores-.

(36) ¡Mi Lopico eres, que bien lo sé, aunque no te veo! ;En los olores te conozco, que tú eres mi Lope! (Miras, D. 1992. Las brujas de Barahona. Madrid: Espasa Calpe).

Sea como fuere, parece claro que en la base de los predicados de conocer hay siempre una evidencia que manifiesta la relación con la fuente que es origen del conocimiento, y la TBS expresa esa evidencia por medio de la relación entre un presupuesto relativo al contacto con la fuente del conocimiento (lo que representamos como "haber visto/olido/escuchado/...") y el conocimiento efectivo, algo que se formaliza en una $\mathrm{AE}$. 


\subsection{La evidencialidad en otros predicados estativos}

Si nuestra hipótesis es correcta, otros predicados estativos deberían responder a la misma condición que hemos constatado en estar y en conocer. Y, en efecto, a otros predicados estativos se les asocian, en una $\mathrm{AE}$, discursos que manifiestan alguna forma de evidencialidad.

Así, para el caso de resulta que, puede verse (37), donde resulta que admite ser el aporte (o segmento a la derecha) de un encadenamiento argumentativo que presenta a la izquierda del conector un predicado evidencial, encadenamiento que puede concebirse como una de las $\mathrm{AE}$ estructurales de resulta que.

(37) De acuerdo con diversas pruebas de laboratorio, las mujeres suelen aventajar a los hombres en agudeza auditiva, y además la conservan plena durante más años que ellos. Es probable que nos encontremos ante una nueva jugarreta del entorno cultural. El mismo fenómeno parece agazapado en los dominios del arte culinario, que nos suministra otro ejemplo interesante. Hombres son, sin excepción, los cocineros célebres, pero ahora resulta que la mujer tiene, por término medio, un sentido del gusto más fino (Penella, M. 1995. Tu hijo: genio en potencia. Las claves fundamentales para su educación. Madrid: Espasa Calpe).

De hecho, el enunciado de (37) puede formalizarse como las evidencias científicas (pruebas de laboratorio) muestran $p$ PLT resulta que $p$, y el segmento a la izquierda puede generalizarse como un predicado evidencial del tipo se ve/constata que p. Más aún, siempre parece requerirse una constatación previa (sea esta una evidencia directa o una evidencia indirecta) para que se admita el enunciado de resulta que.

Del mismo modo, para el caso de querer, puede decirse que una de sus AE a la izquierda se concreta con predicados que acreditan el reconocimiento de la carencia o la necesidad como, por ejemplo, el que muestra (38).

(38) Tengo hambre, quiero comer. Tengo un sandwich en mi bolsillo (Ocampo, S. 1988. Cornelia frente al espejo. Barcelona: Tusquets).

En efecto, en (38), querer es el aporte o segmento a la derecha de un argumento en el que la necesidad funciona como presupuesto, al modo de tiene necesidad de X PLT quiere $X$, un caso de evidencia directa endofórica en los términos de Bermúdez (ver más arriba en $\$$ La evidencialidad). $\mathrm{O}$ incluso lo que se propone en (39), cuyo presupuesto -o segmento a la izquierda- es un discurso que evoca lo que Bermúdez llama evidencia directa sensorial (en este caso, un predicado de ver). 
(39) Llevo leyendo blogs desde hará unos ocho meses, y con la idea de hacer uno propio (ya sabéis, culo veo culo quiero) desde hace como mes y medio, mes y medio en el que, en ratos libres, he ido pensando y probando mil diseños para mi blog (Efímero. 02206003. España. Página web 2002) ${ }^{11}$.

Concretamente, la frase cristalizada que destacamos en (39) no es más que la muestra palmaria de la asociación entre un discurso evidencial (que expresa la fuente del conocimiento) y el predicado de querer.

En definitiva, la existencia de una expresión evidencial como formante de una de las AE de estos predicados estativos manifiesta su conexión con la evidencialidad. Pero no sólo eso. Al mismo tiempo, y en conjunción con otras condiciones, los agrupa en una subcategoría dentro de los estativos, categoría que venimos denominando estativos delimitados.

Como hemos mostrado en otros lugares según su comportamiento en vistas de la externalización (Ramírez Gelbes, 2008a), de los falsos infinitivos y los falsos participios (Ramírez Gelbes, 2008b), de la correferencia (Ramírez Gelbes, 2008c), de los gerundios (Ramírez Gelbes, 2008d) o de los modificadores de alto grado (Ramírez Gelbes, 2011), por ejemplo, los predicados desarrollados por estar + adjetivo, por estar en, por conocer, por resulta que y por querer se distinguen de otros (como ser, haber, saber, parece que o gustar) que hemos llamado puros y manifiestan, a su vez, comportamientos similares dentro de su grupo. Y es por ese motivo que, sostenemos, la presencia de un contenido evidencial en una de las $\mathrm{AE}$ estructurales de un predicado estativo es una prueba que orienta a su inclusión dentro del grupo de los estativos delimitados.

\section{A MANERA DE CONCLUSIÓN}

En este trabajo hemos intentado demostrar la relación entre algunos estativos -específicamente, estar + adjetivo, estar en, conocer, resulta que y querer- y la evidencialidad. A la luz de los supuestos de la Teoría de los Bloques Semánticos, hemos observado que al menos una de las argumentaciones externas estructurales de estos estativos -a los que, por otra parte, venimos llamando estativos delimitados- contiene, de uno de los lados del conector, la expresión estativa y, del otro, una expresión evidencial. La presencia de esa expresión evidencial se une a otras condiciones que exhiben esos mismos estativos y nos permite considerarla una prueba más para reconocer miembros de ese grupo, esto es, de estativos que perte-

${ }^{11}$ Lo sabemos, también es posible postular veo PLT Neg-quiero, lo que muestra que este caso no constituye una $\mathrm{AE}$ estructural sino contextual. Con todo, la existencia en la lengua de discursos cristalizados que establecen una relación normativa entre el ver y el querer parece patentizar esta relación de los predicados de querer con la evidencialidad. 
necen a la subclase de los delimitados. Queda, para la investigación futura, la tarea de identificar otros predicados estativos que pertenezcan a esta misma subclase.

\section{REFERENCIAS}

Aikhenvald, A. Y. (2004). Evidentiality. Oxford: Oxford University Press.

Alonso, A. y P. Henríquez Ureña. (1946). Gramática castellana. Buenos Aires, Losada.

Anscombre, J.C. y O. Ducrot. (1983). L'argumentation dans la langue. Bruxelles: Pierre Mardaga.

Anscombre, J.C. y O. Ducrot. (1994). La argumentación en la lengua. Madrid: Gredos.

Bach, E. (1986). The algebra of events. Linguistics and Philosophy, 9, 5-16.

Bello, A. (1945) [1847]. Gramática de la lengua castellana destinada al uso de los americanos. Buenos Aires: Sopena.

Bermúdez, F. (2005). Evidencialidad. La codificación del punto de vista. Tesis Doctoral, Universidad de Estocolmo.

Bruxelles, S., O. Ducrot y P.Y. Raccah. (1993). Argumentation et champs topiques lexicaux. Cahiers de Praxématique, 21, 88-104.

Carel, Marion (1992). Vers une formalisation de la théorie de l'argumentation dans la langue. Tesis Doctoral, Paris: EHESS.

Chafe, W.L. y J. Nichols (eds.). (1986). Evidentiality: The linguistic encoding of epistemology. Norwood, NJ: Ablex.

Comrie, B. (1976). Aspect. An introduction to the study of verbal aspect and related problems. Cambridge: CUP.

Dowty, D. (1979). Word meaning and Montague grammar. The semantics of verbs and times in Generative Semantics and in Montague's PTQ. Dordrecht: Reidel.

Egg, M. (1995). The intergressive as a new category of verbal Aktionsart. Journal of Semantics, 12, 311-356.

Falk, J. (1979). Visión de norma general vs. visión de norma individual. Ensayo de explicación de la oposición serlestar en unión con adjetivos que denotan belleza y corpulencia. Studia Neophilologica, vol. LI, 2, 273-293.

Filip, H. (2005). On accumulating and having it all. En Verkuyl, H., H. de Swart y A. van Hout (eds.), Perspectives on Aspect (pp.125-148). Dordrecht: Springer.

Gili y Gaya, S. (1948). Curso superior de sintaxis española. Barcelona: Spes.

González, P. (2003). Aspects on aspect. Theory and application of grammatical aspect in Spanish. Utrecht: LOT.

Guentchéva, Zlatka (Ed.). (1996). L'Énonciation médiatisée. Bibliothèque de l'information grammaticale. Louvain: Éditions Peeters.

Hinrichs, E. (1986). A compositional semantics for Aktionsarten and NP-referen- 
ce in English. Tesis Doctoral, Ohio State University, Columbus.

López Ferrero, C. (2005) Funciones retóricas en la comunicación académica: formas léxicas de modalidad y evidencialidad. Signo y Seña, 23, 115-139.

Lyons, J. (1997). Semántica lingüistica. Barcelona: Paidós.

Marín, R. (2000). El componente aspectual de la predicación. Tesis Doctoral, Universidad Autónoma de Barcelona.

Matte Bon, F. (1995). Gramática comunicativa del español (nueva edición revisada). Madrid: Edelsa.

Miguel Aparicio, E. (1999). El aspecto léxico. En Bosque, I. y V. Demonte (eds), Gramática descriptiva de la lengua española (pp. 2977-3060). Madrid: Espasa Calpe.

Morimoto, Y. (1998). El aspecto léxico: delimitación. Madrid: Arco/Libros.

Mourelatos, A. (1978). Events, processes, and states. Linguistics and Philosophy, 2, 415-434.

Porroche Ballesteros, M. (1988). Ser, estar y verbos de cambio. Madrid: Arco/Libros.

Ragucci, R. (1956). El habla de mi tierra. Buenos Aires: Editorial Don Bosco.

Ramírez Gelbes, S. (2004). Ser y estar y la aspectualidad léxica: un caso de restricción procedimental. Tesis de maestría inédita. Madrid: UNED.

Ramírez Gelbes, S. (2008a). La Teoría de los Bloques Semánticos y la aspectualidad: los 'externalizados'. Analecta Malacitana, 25, pp. 81-105. Disponible en http://www.anmal.uma.es/numero25/Gelbes.pd.

Ramírez Gelbes, S. (2008b). Los participios estativos que se usan como sustantivos: de cómo un estado puede ser un resultado. Ponencia presentada en el XV Congreso internacional de la Asociación de Lingüística y Filología de América Latina, Universidad de la República: Montevideo, Uruguay.

Ramírez Gelbes, S. (2008c). Correferencia y aspectualidad. La correferencia inclusiva y los verbos télicos. Zeitschrift für romanische Philologie, 124, 514-530.

Ramírez Gelbes, S. (2008d). Gerundios y aspectualidad: acerca de los estativos. Romanische Forschungen, 120, 2, 143-165.

Ramírez Gelbes, S. (2010). Semántica argumentativa y aspectualidad: estativos puros y estativos delimitados. El caso de los predicados de ser/estar y saber/ conocer. Tesis Doctoral, Universidad de Buenos Aires.

Ramírez Gelbes, S. (2011). Aspectualidad, modificadores de alto grado y la Teoría de los Bloques Semánticos: un análisis de hasta y como aplicados a estativos. ALH, XXVI, 65-88.

Rémi-Giraud, S. y M. Le Guern (dirs.) (1986). Sur le verbe. Lyon: Presses Universitaires de Lyon.

Reyes, G. (1994). Los procedimientos de cita: Citas encubiertas y ecos. Madrid: Arco/ Libros.

Saussure, F. de (1955). Curso de lingüistica general. Buenos Aires: Losada. 
Tenny, C. (1989). The aspectual interface hypothesis. NELS, 18, 1-27.

Vendler, Z. (1967). Verbs and times. En Z. Vendler, Linguistics in philosophy (pp. 97-121). Ithaca: Cornell University Press.

Verkuyl, H. (1993). A theory of aspectuality. The Interaction between temporal and atemporal structure. Cambridge: CUP.

Willet, T. (1988). A cross-linguistic survey of the grammaticalization of evidentiality. Studies in Language, 12, 51-97. 